\title{
A Nonlocal Model for Carbon Nanotubes under Axial Loads
}

\author{
Raffaele Barretta and Francesco Marotti de Sciarra \\ Department of Structures for Engineering and Architecture, University of Naples Federico II, Via Claudio 21, 80125 Naples, Italy \\ Correspondence should be addressed to Raffaele Barretta; rabarret@unina.it
}

Received 7 July 2013; Revised 8 October 2013; Accepted 10 October 2013

Academic Editor: Jun Liu

Copyright (C) 2013 R. Barretta and F. Marotti de Sciarra. This is an open access article distributed under the Creative Commons Attribution License, which permits unrestricted use, distribution, and reproduction in any medium, provided the original work is properly cited.

\begin{abstract}
Various beam theories are formulated in literature using the nonlocal differential constitutive relation proposed by Eringen. A new variational framework is derived in the present paper by following a consistent thermodynamic approach based on a nonlocal constitutive law of gradient-type. Contrary to the results obtained by Eringen, the new model exhibits the nonlocality effect also for constant axial load distributions. The treatment can be adopted to get new benchmarks for numerical analyses.
\end{abstract}

\section{Introduction}

Carbon nanotubes (CNTs) are a topic of major interest both from theoretical and applicative points of view. This subject is widely investigated in literature to describe small-scale effects [1-4], vibration and buckling [5-13], and nonlocal finite element analysis [14-18]. A comprehensive review on applications of nonlocal elastic models for CNTs is reported in [19] and therein references. Buckling of triple-walled CNTs under temperature fields is dealt with in [20]. An alternative methodology is based on an atomistic-based approach [21] which predicts the positions of atoms in terms of interactive forces and boundary conditions. The standard approach to analyze CNTs under axial loads consists in solving an inhomogeneous second-order ordinary differential equation providing the axial displacement field, see, for example, [22]. The known term of the differential equation is the sum of two contributions. The former describes the local effects linearly depending on the axial load. The latter characterizes the small-scale effects depending linearly on the second derivative along the rod axis of the axial load. This model is thus not able to evaluate small-scale effects due to, constant axial loads per unit length. This approach, commonly adopted in literature, is based on the following nonlocal linearly elastic constitutive law proposed by Eringen [23]:

$$
\sigma-e_{o}^{2} a^{2} \sigma^{(2)}=E \varepsilon
$$

where $e_{o}$ is a material constant, $a$ is the internal length, $E$ is the Young modulus, $\sigma$ is the normal stress, the apex $(\bullet)^{(2)}$ is second derivative along the rod axis, and $\varepsilon$ is the axial elongation. Indeed, integrating on the rod cross section domain $\Omega$ and imposing that the axial force $N$ is equal to the resultant of normal stress field we get the differential equation

$$
N-e_{o}^{2} a^{2} N^{(2)}=E A w^{(1)},
$$

where $\varepsilon=w^{(1)}$, with $w^{(1)}$ being first derivative along the rod axis of the axial displacement field $w:[0, L] \mapsto \mathscr{R}$, where $L$ is the rod length and $A$ denotes the cross section area. Since the equilibrium prescribes that the first derivative of $N$ is opposite to the axial load $p$, we infer the well-known differential equation (see, e.g., [7]) as follows:

$$
w^{(2)}=\frac{-p+e_{o}^{2} a^{2} p^{(2)}}{E A} .
$$

Note that the nonlocal contribution vanishes for constant loads $p$. In the present paper, an alternative nonlocal constitutive behavior is adopted to assess small-scale effects in nanotubes also for constant axial loads. The corresponding axial displacement field is shown to be governed by a fourth-order inhomogeneous differential equation. Boundary conditions are naturally inferred by performing a standard localization procedure of a variational problem formulated by making recourse to thermodynamic restrictions see, for example, 
[24-26], according to the geometric approach illustrated in [27-30]. As an example, the displacement field of nanotubes under constant axial loads per unit length is evaluated in the appendix. Vibration and buckling effects are not the subject of this paper and will be addressed in a forthcoming paper.

\section{Nonlocal Variational Formulation}

Let $\mathscr{B}$ be the three-dimensional spatial domain of a straight rod subjected to axial loads. An apex $(\bullet)^{(n)}$ stands for $n$th derivative along the rod centroidal $z$-axis. Kinematic compatibility between axial elongations $\varepsilon$ and axial displacements $w$ is expressed by the differential equation $\varepsilon=w^{(1)}$. Denoting by a dot the time-rate, the following noteworthy relations hold true

$$
\dot{\varepsilon}=\dot{w}^{(1)}, \quad \dot{\varepsilon}^{(1)}=\dot{w}^{(2)} .
$$

The differential equation of equilibrium turns out to be $N^{(1)}=$ $-p$. Boundary equilibrium prescribes that at the end cross sections act axial loads equal to $N(0)$ for $z=0$ and to $N(L)$ for $z=L$. Let us now consider a nonlocal constitutive model of gradient-type defined by assigning the following elastic energy functional per unit volume:

$$
\psi\left(\varepsilon, \varepsilon^{(1)}\right):=\frac{1}{2} E \varepsilon^{2}+\frac{1}{2} E c^{2} \varepsilon^{(1)^{2}}
$$

with $c:=e_{o} a$ being nonlocal parameter. Relation (5) is similar to the elastic energy density proposed in [31] where a homogeneous quadratic functional including also mixed terms is assumed. The elastic energy time rate is hence expressed by the formula

$$
\dot{\psi}\left(\varepsilon, \varepsilon^{(1)}\right)=\frac{\partial \psi}{\partial \varepsilon} \dot{\varepsilon}+\frac{\partial \psi}{\partial \varepsilon^{(1)}} \dot{\varepsilon}^{(1)}=\sigma_{o} \dot{\varepsilon}+\sigma_{1} \dot{\varepsilon}^{(1)}
$$

where

$$
\sigma_{o}:=\frac{\partial \psi}{\partial \varepsilon}=E \varepsilon, \quad \sigma_{1}:=\frac{\partial \psi}{\partial \varepsilon^{(1)}}=E c^{2} \varepsilon^{(1)}
$$

are the static variables conjugating with the kinematic variables $\varepsilon$ and $\varepsilon^{(1)}$. The static variable $\sigma_{1}$ is the scalar counterpart of the so-called double stress tensor [31]. By imposing the thermodynamic condition (see, e.g., [32-34])

$$
\int_{\mathscr{B}} \sigma \dot{\varepsilon} d V-\int_{\mathscr{B}} \dot{\psi} d V=0
$$

where $\sigma$ is the normal stress, we infer the relation

$$
\int_{\mathscr{B}} \sigma \dot{\varepsilon} d V=\int_{\mathscr{B}} \sigma_{o} \dot{\varepsilon} d V+\int_{\mathscr{B}} \sigma_{1} \dot{\varepsilon}^{(1)} d V
$$

The relevant differential and boundary equations are thus obtained as shown hereafter. Substituting the expression of the rates $\dot{\varepsilon}$ and $\dot{\varepsilon}^{(1)}$ in terms of the axial displacement $w(z)$ of the cross section at abscissa $z$, we get the formulae

$$
\begin{aligned}
\int_{\mathscr{B}} \sigma \dot{\varepsilon} d V & =\int_{\mathscr{B}} \sigma \dot{w}^{(1)} d V=\int_{0}^{L}\left(\int_{\Omega} \sigma d A\right) \dot{w}^{(1)} d z \\
& =\int_{0}^{L} N \dot{w}^{(1)} d z, \\
\int_{\mathscr{B}} \sigma_{o} \dot{\varepsilon} d V= & \int_{0}^{L} N_{o} \dot{w}^{(1)} d z, \\
\int_{\mathscr{B}} \sigma_{1} \dot{\varepsilon}^{(1)} d V & =\int_{\mathscr{B}} \sigma_{1} \dot{w}^{(2)} d V=\int_{0}^{L}\left(\int_{\Omega} \sigma_{1} d A\right) \dot{w}^{(2)} d z \\
& =\int_{0}^{L} N_{1} \dot{w}^{(2)} d z,
\end{aligned}
$$

with $N=\int_{\Omega} \sigma d A$ axial force (static equivalence condition on the cross sections) and $N_{i}:=\int_{\Omega} \sigma_{i} d A$ for $i \in\{0,1\}$. Thermodynamic condition (9) provides the axial contribution

$$
\int_{0}^{L} N \dot{w}^{(1)} d z=\int_{0}^{L} N_{o} \dot{w}^{(1)} d z+\int_{0}^{L} N_{1} \dot{w}^{(2)} d z
$$

\section{Differential and Boundary Equations of Elastic Equilibrium}

Resorting to Green's formula, a standard localization procedure provides the differential and boundary equations corresponding to the variational conditions inferred in Section 2, as follows. A direct computation gives

$$
\begin{gathered}
\int_{0}^{L} N \dot{w}^{(1)} d z=[N \quad \dot{w}]_{0}^{L}-\int_{0}^{L} N^{(1)} \dot{w} d z \\
\int_{0}^{L} N_{o} \dot{w}^{(1)} d z=\left[N_{o} \dot{w}\right]_{0}^{L}-\int_{0}^{L} N_{o}^{(1)} \dot{w} d z, \\
\int_{0}^{L} N_{1} \dot{w}^{(2)} d z=\left[N_{1} \dot{w}^{(1)}\right]_{0}^{L}-\int_{0}^{L} N_{1}^{(1)} \dot{w}^{(1)} d z \\
=\left[N_{1} \dot{w}^{(1)}\right]_{0}^{L}-\left[N_{1}^{(1)} \dot{w}\right]_{0}^{L}+\int_{0}^{L} N_{1}^{(2)} \dot{w} d z .
\end{gathered}
$$

Substituting into the variational condition (11), a suitable localization provides the relevant differential equation

$$
N^{(1)}=N_{o}^{(1)}-N_{1}^{(2)}
$$

and boundary conditions

$$
\begin{aligned}
& N=N_{o}-N_{1}^{(1)}, \quad \text { dual of } \dot{w}, \\
& 0=N_{1}, \quad \text { dual of } \dot{w}^{(1)} .
\end{aligned}
$$


These conditions can be conveniently expressed in terms of the axial displacement field $w$ as follows. A direct evaluation of the scalar functions $N_{i}:[0, L] \mapsto \mathscr{R}$ for $i \in\{0,1\}$ and of their derivatives gives

$$
\begin{gathered}
N_{o}=\int_{\Omega} \sigma_{o} d A=\int_{\Omega} E \varepsilon d A=\int_{\Omega} E w^{(1)} d A=E A w^{(1)}, \\
N_{o}^{(j)}=E A w^{(1+j)} \\
N_{1}=\int_{\Omega} \sigma_{1} d A=\int_{\Omega} E c^{2} \varepsilon^{(1)} d A \\
=\int_{\Omega} E c^{2} w^{(2)} d A=E A c^{2} w^{(2)} \\
N_{1}^{(j)}=E A c^{2} w^{(2+j)}
\end{gathered}
$$

with $j \in\{1,2, \ldots, n\}$. Accordingly, the boundary and differential conditions of elastic equilibrium (13) and (14) take the form

$$
\begin{gathered}
N^{(1)}=N_{o}^{(1)}-N_{1}^{(2)}=E A w^{(2)}-E A c^{2} w^{(4)}, \\
N=N_{o}-N_{1}^{(1)}=E A w^{(1)}-E A c^{2} w^{(3)}, \quad \text { dual of } \dot{w}, \\
0=N_{1}=E A c^{2} w^{(2)}, \quad \text { dual of } \dot{w}^{(1)} .
\end{gathered}
$$

\section{Example}

Let us consider a straight rod subject to a constant axial load $p$ as depicted in Figure 1. End cross sections $\mathbf{A}$ and $\mathbf{B}$ are assumed to be hinged and simply supported, respectively. As illustrated in Section 3, the computation of the rod axial displacement field $w$ involves the following cross section geometric and elastic properties: area $A$, Young modulus $E$, and nonlocal parameter $c$. By setting $\alpha:=E A c^{2}$ and $\beta:=E A$, the differential equation of elastic equilibrium is as follows:

$$
\alpha w^{(4)}-\beta w^{(2)}=p .
$$

The general integral takes thus the form (see the appendix)

$$
w(z)=w_{H}(z)+\bar{w}(z),
$$

with

$$
\begin{aligned}
w_{H}(z)= & c_{1}+c_{2} z+c_{3} \exp \left(\sqrt{\left.\frac{\beta}{\alpha} z\right)}\right. \\
& +c_{4} \exp \left(-\sqrt{\left.\frac{\beta}{\alpha} z\right)},\right. \\
& \bar{w}(z)=c_{5} z^{2} .
\end{aligned}
$$

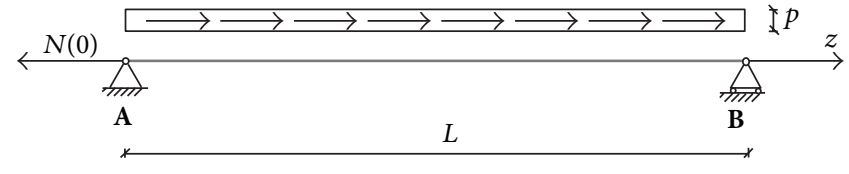

FIgURE 1: Rod under constant axial load.

The evaluation of the constants is carried out by imposing the following boundary conditions (see also Section 3):

$$
\begin{gathered}
w(0)=0, \\
N(L)=E A w^{(1)}(L)-E A c^{2} w^{(3)}(L)=0, \\
N_{1}(0)=E A c^{2} w^{(2)}(0)=0, \\
N_{1}(L)=E A c^{2} w^{(2)}(L)=0, \\
\mathbb{\mathbb { }} \\
w(0)=0, \\
w^{(1)}(L)-c^{2} w^{(3)}(L)=0, \\
w^{(2)}(0)=0, \\
w^{(2)}(L)=0 .
\end{gathered}
$$

Resorting to the expressions of the derivatives $w_{H}^{(j)}$ and $\bar{w}^{(j)}$ for $j \in\{1,2,3,4\}$,

$$
\begin{aligned}
& w_{H}^{(1)}(z)=c_{2}+\frac{c_{3}}{c} \exp \left(\frac{1}{c} z\right)-\frac{c_{4}}{c} \exp \left(-\frac{1}{c} z\right), \\
& w_{H}^{(2)}(z)=\frac{c_{3}}{c^{2}} \exp \left(\frac{1}{c} z\right)+\frac{c_{4}}{c^{2}} \exp \left(-\frac{1}{c} z\right), \\
& w_{H}^{(3)}(z)=\frac{c_{3}}{c^{3}} \exp \left(\frac{1}{c} z\right)-\frac{c_{4}}{c^{3}} \exp \left(-\frac{1}{c} z\right), \\
& w_{H}^{(4)}(z)=\frac{c_{3}}{c^{4}} \exp \left(\frac{1}{c} z\right)+\frac{c_{4}}{c^{4}} \exp \left(-\frac{1}{c} z\right), \\
& \bar{w}^{(1)}(z)=2 c_{5} z \\
& \bar{w}^{(2)}(z)=2 c_{5} \\
& \bar{w}^{(3)}(z)=0 \\
& \bar{w}^{(4)}(z)=0
\end{aligned}
$$

and having $\sqrt{\beta / \alpha}=1 / c$, a direct computation provides the algebraic system

$$
\begin{gathered}
c_{1}+c_{3}+c_{4}=0 \\
c_{2}+2 L c_{5}=0 \\
\frac{1}{c^{2}} c_{3}+\frac{1}{c^{2}} c_{4}+2 c_{5}=0 \\
\frac{1}{c^{2}} \exp \left(\frac{1}{c} L\right) c_{3}+\frac{1}{c^{2}} \exp \left(-\frac{1}{c} L\right) c_{4}+2 c_{5}=0 .
\end{gathered}
$$




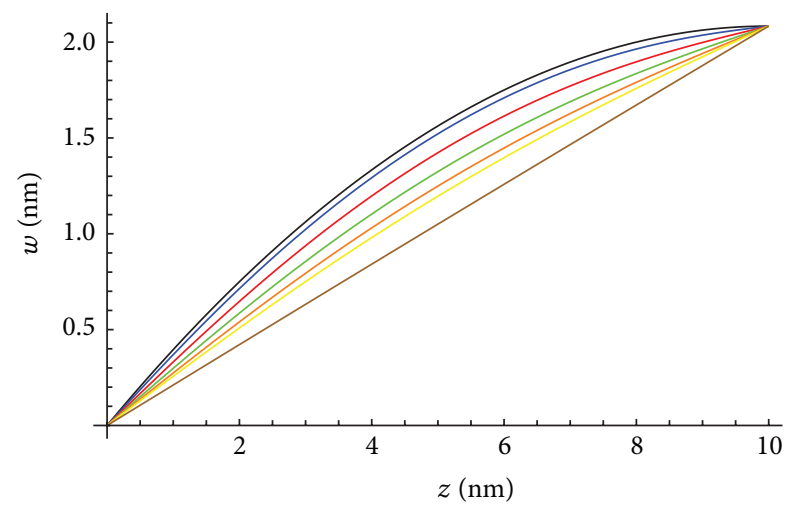

Figure 2: Axial displacement field $w$ for $c=0$ (local solution-black line), $c=1 \mathrm{~nm}$ (blue line), $c=2 \mathrm{~nm}$ (red line), $c=3 \mathrm{~nm}$ (green line), $c=4 \mathrm{~nm}$ (orange line), $c=5 \mathrm{~nm}$ (yellow line), and $c=25 \mathrm{~nm}$ (brown line). $E=300 \mathrm{GPa} ; L=10 \mathrm{~nm} ; A=80 \cdot 10^{-2} \mathrm{~nm}^{2}$; and $p=10^{-8} \mathrm{~N} / \mathrm{nm}$.

A further condition can be obtained by imposing that the scalar field

$$
\bar{w}(z)=c_{5} z^{2}
$$

is a particular solution of the differential equation (17), whence it follows that $c_{5}=-p / 2 E A$. The remaining constants are given by the formulae

$$
\begin{gathered}
c_{1}=-\left(c_{3}+c_{4}\right), \\
c_{2}=-2 L c_{5}, \\
c_{3}=\frac{2 c^{2}(1-\exp (-(1 / c) L))}{\exp (-(1 / c) L)-\exp ((1 / c) L)} c_{5}, \\
c_{4}=\frac{2 c^{2}(1-\exp ((1 / c) L))}{\exp ((1 / c) L)-\exp (-(1 / c) L)} c_{5},
\end{gathered}
$$

having $c_{5}=-p / 2 E A$. A plot of the rod axial displacement field $w$ for different values of the nonlocal parameter $c$ is provided in Figure 2. It is apparent that the rod becomes stiffer if the nonlocal parameter increases. The evaluated axial displacement at the free end of the rod $\mathbf{B}$ provides the same value independently of the nonlocal parameter. Such a value coincides with the displacement of the point $\mathbf{B}$ if a local model is considered. Moreover, the limit of the axial displacement field for $c$ tending to plus infinity can be evaluated to get the lower bound

$$
w^{\text {low }}(z):=\lim _{c \rightarrow+\infty} w(z, c)=0.208333 z
$$

Hence, large values of the nonlocal parameter provide a displacement field which tends to a linear one, see Figure 2, for $c=25$. Further, the limit value of the axial displacement for $z=L$ and $c \rightarrow+\infty$, obtained by (25), yields $w^{\text {low }}(L)=$ $2.08333 \mathrm{~nm}$ which coincides with the axial displacement at $\mathbf{B}$ for any value of the nonlocal parameter $c$, see Figure 3 and Table 1.

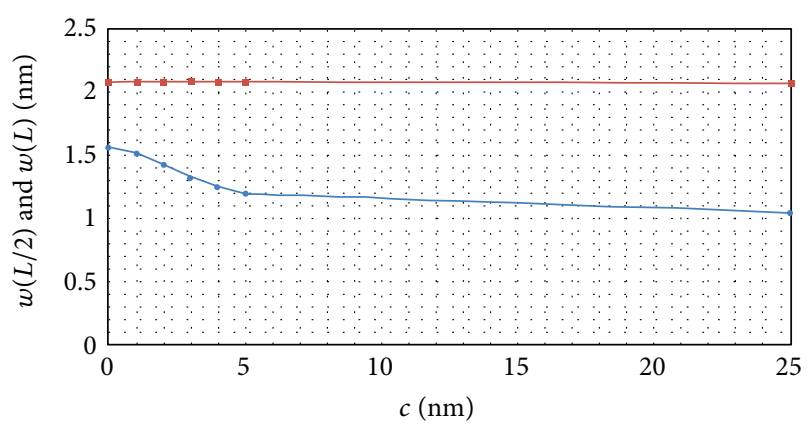

FIGURE 3: Axial displacement in terms of the nonlocal parameter $c$ at the abscissa $z=L / 2$ (blue line) and $z=L$ (red line).

TABLE 1: Axial displacements $w(L / 2)$ and $w(L)$ versus the nonlocal parameter $c$.

\begin{tabular}{lcc}
\hline$c(\mathrm{~nm})$ & $w(L / 2)(\mathrm{nm})$ & $w(L)(\mathrm{nm})$ \\
\hline 0 & 1.5625 & 2.08333 \\
1 & 1.52139 & 2.08333 \\
2 & 1.42301 & 2.08333 \\
3 & 1.32428 & 2.08333 \\
4 & 1.24886 & 2.08333 \\
5 & 1.19589 & 2.08333 \\
25 & 1.05021 & 2.08333 \\
\hline
\end{tabular}

The upper bound of the axial displacement is provided by the local solution (i.e., $c=0$ )

$$
w^{\text {upp }}(z):=w(z, 0)=\frac{p(z)(2 L-z) z}{2 E A} .
$$

The axial displacement evaluated for $z=L$ by (26) yields the value $w^{\text {upp }}(L)=25 / 12 \mathrm{~nm}$ which coincides with the axial displacement at $\mathbf{B}$ for any value of $c$, see Figure 3 and Table 1. For the considered model, the upper and lower bounds of the axial displacement field are given by (25) and (26). The axial displacement $w(L / 2)$ at the middle point of the rod and the maximum axial displacement $w(L)$ as functions of the nonlocal parameter $c$ are depicted in Figure 3. The corresponding numerical values of $w(L / 2)$ and $w(L)$ are listed in Table 1.

It is worth noting that equilibrium prescribes that axial force $N$ must be a linear function, confirmed by the blue diagram in Figure 4 obtained as difference between the local contribution $N_{o}$ (dashed line) and the nonlocal one $N_{1}^{(1)}$ (continuous thin line), according to (14) for any value of $c$.

\section{Conclusions}

The outcomes of the present paper may be summarized as follows.

(i) Linearly elastic carbon nanotubes under axial loads have been investigated by a nonlocal variational approach based on thermodynamic restrictions. The treatment provides an effective tool to evaluate smallscale effects in nanotubes subject also to constant 


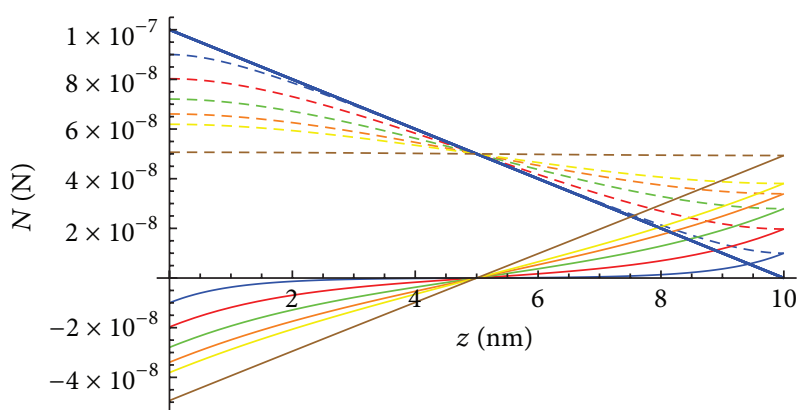

Figure 4: Axial force $N=N_{o}-N_{1}^{(1)}$ (blue line), $N_{o}$ (dashed line), $N_{1}^{(1)}$ (continuous thin line); $c=0$ (local solution-black line), $c=$ $1 \mathrm{~nm}$ (blue line), $c=2 \mathrm{~nm}$ (red line), $c=3 \mathrm{~nm}$ (green line), $c=4 \mathrm{~nm}$ (orange line), $c=5 \mathrm{~nm}$ (yellow line), and $c=25 \mathrm{~nm}$ (brown line).

axial loads, a goal not achievable by the Eringen model commonly adopted in literature as motivated in Section 1.

(ii) Relevant boundary and differential conditions of elastic equilibrium have been inferred by a standard localization procedure. Such a procedure provides, in a consistent way, the relevant class of boundary conditions for the nonlocal model.

(iii) The present approach yields a firm thermodynamic procedure to derive different nonlocal models for CNTs by suitable specializations of the elastic energy.

(iv) Exact solutions of carbon nanotubes subject to a constant axial load have been obtained. An advantage of the proposed procedure consists in providing an effective tool to be used as a benchmark for numerical analyses. Finally, a range to which any nonlocal solution must belong is analytically evaluated.

\section{Appendix}

The procedure to solve the ordinary differential equation

$$
\alpha w^{(4)}-\beta w^{(2)}=f,
$$

with $\alpha, \beta>0$ being constant coefficients and $f: I \subseteq \mathscr{R} \mapsto \mathscr{R}$ being a continuous function, is summarized as follows. Let us consider the homogeneous differential equation

$$
\alpha w^{(4)}-\beta w^{(2)}=0
$$

and the relevant characteristic (algebraic) equation $\alpha \lambda^{4}-$ $\beta \lambda^{2}=0$. The roots of the polynomial $\alpha \lambda^{4}-\beta \lambda^{2}$ are $\lambda_{1}=0$ with multiplicity $2, \lambda_{2}=\sqrt{\beta / \alpha}$ with multiplicity 1 and $\lambda_{3}=$ $-\sqrt{\beta / \alpha}$ with multiplicity 1 . The general integral of (A.2) is thus expressed by the formula

$$
\begin{aligned}
w_{H}(z)= & c_{1}+c_{2} z+c_{3} \exp \left(\sqrt{\frac{\beta}{\alpha}} z\right) \\
& +c_{4} \exp \left(-\sqrt{\frac{\beta}{\alpha}} z\right)
\end{aligned}
$$

with exp denoting exponential function and $c_{i} \in \mathscr{R}$ for $i=$ $\{1, \ldots, 4\}$. The general integral of (A.1) is writen therefore as

$$
w(z)=w_{H}(z)+\bar{w}(z)
$$

where $\bar{w}$ is a particular solution of (A.1). It is worth noting that, for $f$ defined by a polynomial $p_{m}$ of degree $m \geq 0$, the solution $\bar{v}$ can be looked for by setting

$$
\bar{w}(z)=z^{2}\left(A_{0}+A_{1} z+\cdots+A_{m} z^{m}\right),
$$

with $A_{i} \in \mathscr{R}$ for $i=\{1, \ldots, m\}$.

\section{Acknowledgments}

The authors were supported by the "Polo delle Scienze e delle Tecnologie," University of Naples Federico II, through the research project FARO. Useful hints and precious comments by anonymous reviewers are also gratefully acknowledged.

\section{References}

[1] Q. Wang and K. M. Liew, "Application of nonlocal continuum mechanics to static analysis of micro- and nano-structures," Physics Letters A, vol. 363, no. 3, pp. 236-242, 2007.

[2] M. Aydogdu, "A general nonlocal beam theory: its application to nanobeam bending, buckling and vibration," Physica E, vol. 41, no. 9, pp. 1651-1655, 2009.

[3] Ö. Civalek and Ç. Demir, "Bending analysis of microtubules using nonlocal Euler-Bernoulli beam theory," Applied Mathematical Modelling, vol. 35, no. 5, pp. 2053-2067, 2011.

[4] M. A. De Rosa and C. Franciosi, "A simple approach to detect the nonlocal effects in the static analysis of Euler-Bernoulli and Timoshenko beams," Mechanics Research Communications, vol. 48, pp. 66-69, 2013.

[5] M. Aydogdu, "Axial vibration analysis of nanorods (carbon nanotubes) embedded in an elastic medium using nonlocal elasticity," Mechanics Research Communications, vol. 41, pp. 3440, 2012.

[6] M. A. Kazemi-Lari, S. A. Fazelzadeh, and E. Ghavanloo, "Nonconservative instability of cantilever carbon nanotubes resting on viscoelastic foundation," Physica E, vol. 44, no. 7-8, pp. 16231630, 2012.

[7] H.-T. Thai and T. P. Vo, "A nonlocal sinusoidal shear deformation beam theory with application to bending, buckling, and vibration of nanobeams," International Journal of Engineering Science, vol. 54, pp. 58-66, 2012.

[8] M. A. Eltaher, S. A. Emam, and F. F. Mahmoud, "Static and stability analysis of nonlocal functionally graded nanobeams," Composite Structures, vol. 96, pp. 82-88, 2013.

[9] S. A. Emam, "A general nonlocal nonlinear model for buckling of nanobeams," Applied Mathematical Modelling, vol. 37, no. 1011, pp. 6929-6939, 2013.

[10] B. Fang, Y.-X. Zhen, C.-P. Zhang, and Y. Tang, "Nonlinear vibration analysis of double-walled carbon nanotubes based on nonlocal elasticity theory," Applied Mathematical Modelling, vol. 37, no. 3, pp. 1096-1107, 2013.

[11] S. A. M. Ghannadpour, B. Mohammadi, and J. Fazilati, "Bending, buckling and vibration problems of nonlocal Euler beams using Ritz method," Composite Structures, vol. 96, pp. 584-589, 2013. 
[12] M. Şimşek and H. H. Yurtcu, "Analytical solutions for bending and buckling of functionally graded nanobeams based on the nonlocal Timoshenko beam theory," Composite Structures, vol. 97, pp. 378-386, 2013.

[13] B. L. Wang and K. F. Wang, "Vibration analysis of embedded nanotubes using nonlocal continuum theory," Composites $B$, vol. 47, pp. 96-101, 2013.

[14] J. K. Phadikar and S. C. Pradhan, "Variational formulation and finite element analysis for nonlocal elastic nanobeams and nanoplates," Computational Materials Science, vol. 49, no. 3, pp. 492-499, 2010.

[15] C. M. C. Roque, A. J. M. Ferreira, and J. N. Reddy, "Analysis of Timoshenko nanobeams with a nonlocal formulation and meshless method," International Journal of Engineering Science, vol. 49, no. 9, pp. 976-984, 2011.

[16] F. F. Mahmoud, M. A. Eltaher, A. E. Alshorbagy, and E. I. Meletis, "Static analysis of nanobeams including surface effects by nonlocal finite elements," Journal of Mechanical Science and Technology, vol. 26, no. 11, pp. 3555-3563, 2012.

[17] S. C. Pradhan, "Nonlocal finite element analysis and small scale effects of CNTs with Timoshenko beam theory," Finite Elements in Analysis and Design, vol. 50, pp. 8-20, 2012.

[18] M. A. Eltaher, A. E. Alshorbagy, and F. F. Mahmoud, "Vibration analysis of Euler-Bernoulli nanobeams by using finite element method," Applied Mathematical Modelling, vol. 37, no. 7, pp. 4787-4797, 2013.

[19] B. Arash and Q. Wang, "A review on the application of nonlocal elastic models in modeling of carbon nanotubes and graphenes," Computational Materials Science, vol. 51, no. 1, pp. 303-313, 2012.

[20] Y. Yan, W. Q. Wang, and L. X. Zhang, "Nonlocal effect on axially compressed buckling of triple-walled carbon nanotubes under temperature field," Applied Mathematical Modelling, vol. 34, no. 11, pp. 3422-3429, 2010.

[21] R. Rafiee and R. M. Moghadam, "On the modeling of carbon nanotubes: a critical review," Composites B, vol. 56, pp. 4354490, 2014

[22] J. N. Reddy, "Nonlocal theories for bending, buckling and vibration of beams," International Journal of Engineering Science, vol. 45, no. 2-8, pp. 288-307, 2007.

[23] A. C. Eringen, "On differential equations of nonlocal elasticity and solutions of screw dislocation and surface waves," Journal of Applied Physics, vol. 54, no. 9, pp. 4703-4710, 1983.

[24] F. Marotti de Sciarra, "Variational formulations, convergence and stability properties in nonlocal elastoplasticity," International Journal of Solids and Structures, vol. 45, no. 7-8, pp. 23222354, 2008.

[25] G. Romano, M. Diaco, and R. Barretta, "Variational formulation of the first principle of continuum thermodynamics," Continuum Mechanics and Thermodynamics, vol. 22, no. 3, pp. 177-187, 2010.

[26] F. Marotti De Sciarra, "Hardening plasticity with nonlocal strain damage," International Journal of Plasticity, vol. 34, pp. 114-138, 2012.

[27] G. Romano and R. Barretta, "Covariant hypo-elasticity," European Journal of Mechanics A, vol. 30, no. 6, pp. 1012-1023, 2011.

[28] G. Romano and R. Barretta, "On Euler's stretching formula in continuum mechanics," Acta Mechanica, vol. 224, no. 1, pp. 211230, 2013.

[29] G. Romano and R. Barretta, "Geometric constitutive theory and frame invariance," International Journal of Non-Linear Mechanics, vol. 51, pp. 75-86, 2013.
[30] G. Romano, R. Barretta, and M. Diaco, "Geometric continuum mechanics," Meccanica, 2013.

[31] R. D. Mindlin, "Micro-structure in linear elasticity," Archive for Rational Mechanics and Analysis, vol. 16, no. 1, pp. 51-78, 1964.

[32] F. Marotti de Sciarra, "Novel variational formulations for nonlocal plasticity," International Journal of Plasticity, vol. 25, no. 2, pp. 302-331, 2009.

[33] F. Marotti de Sciarra, "On non-local and non-homogeneous elastic continua," International Journal of Solids and Structures, vol. 46, no. 3-4, pp. 651-676, 2009.

[34] F. Marotti de Sciarra, "A nonlocal model with strain-based damage," International Journal of Solids and Structures, vol. 46, no. 22-23, pp. 4107-4122, 2009. 

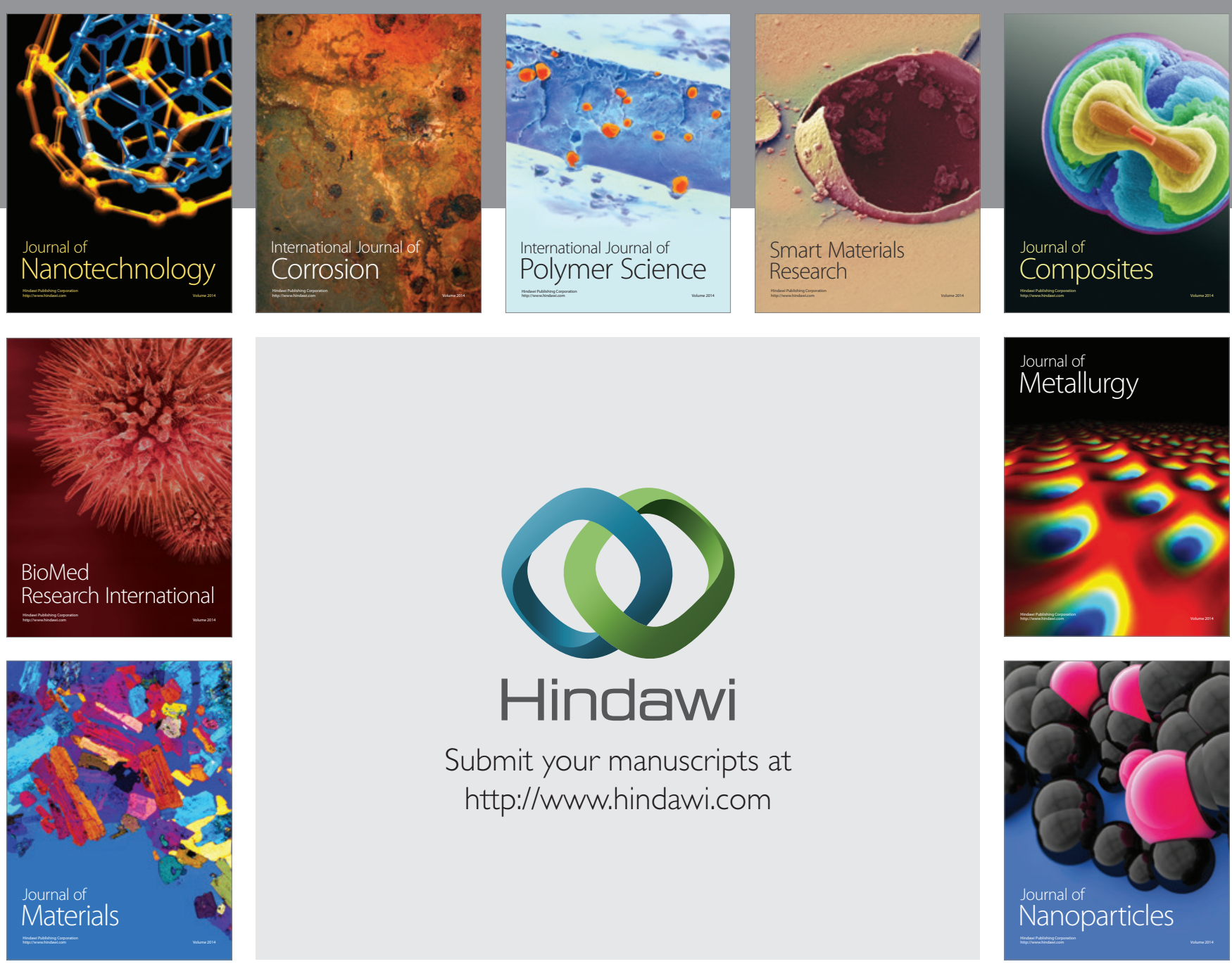

Submit your manuscripts at http://www.hindawi.com
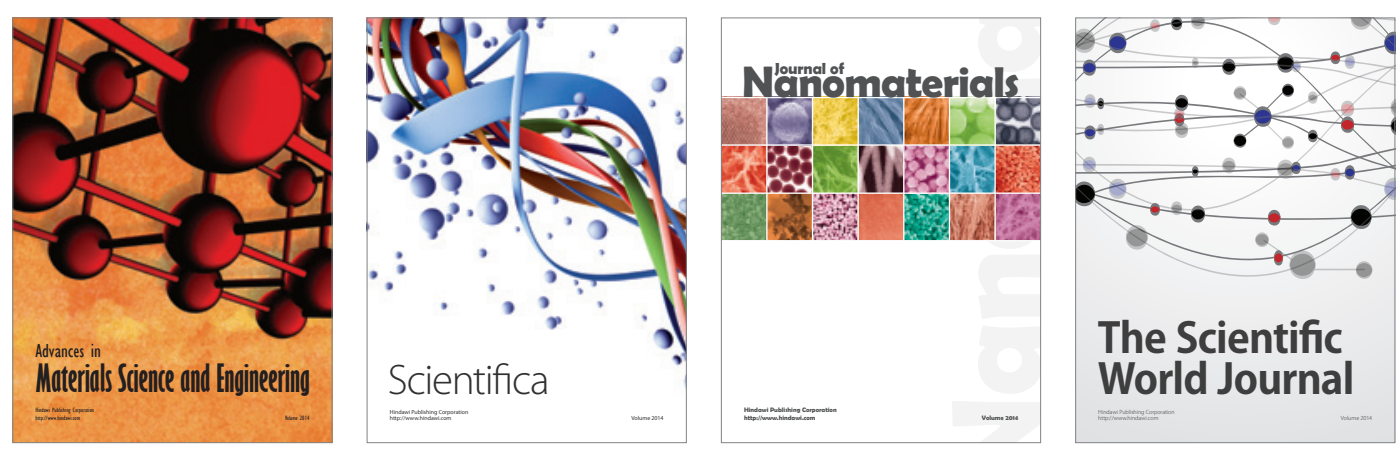

\section{The Scientific World Journal}
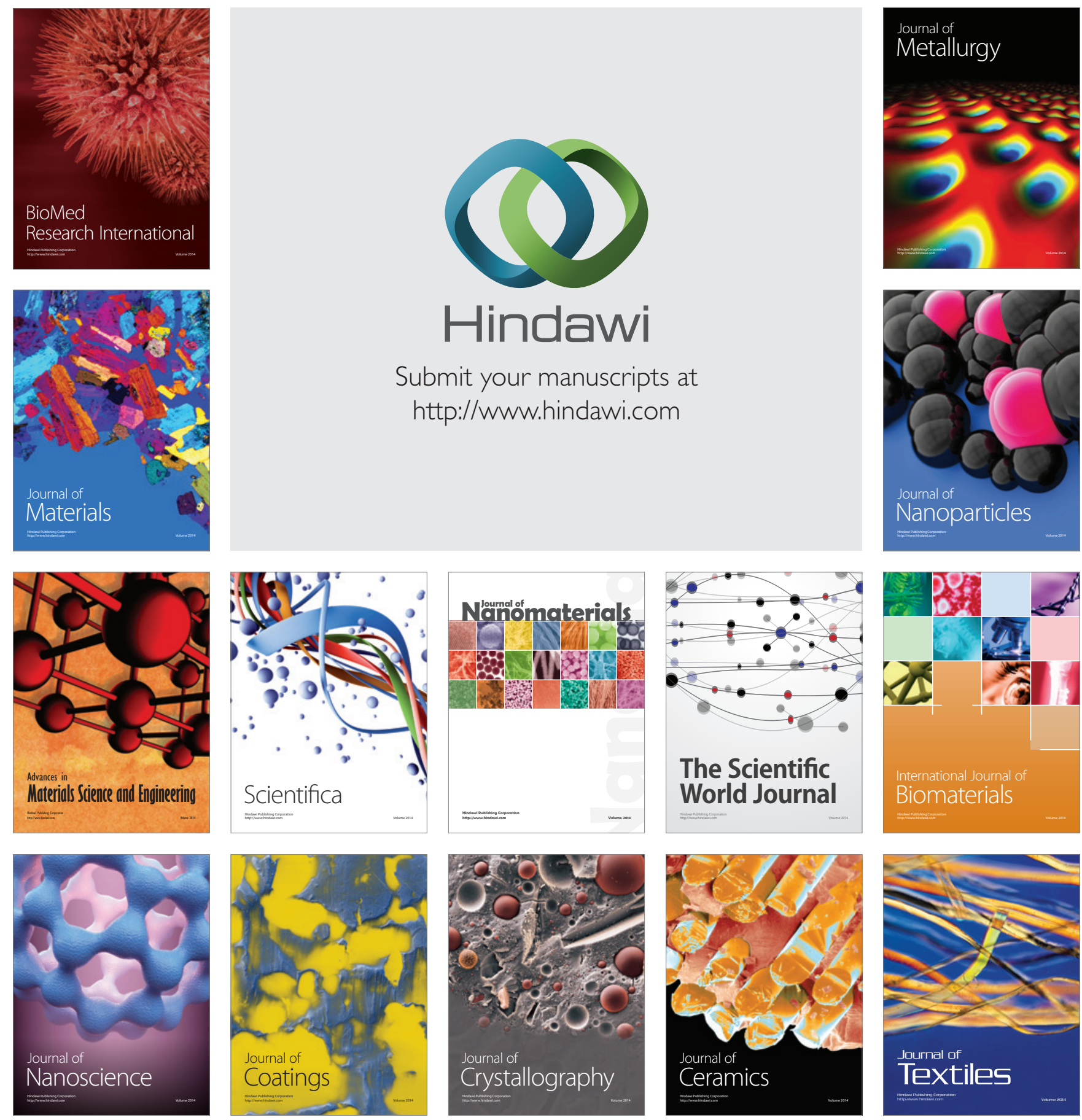